\title{
Self-medication with pilocarpine among outpatients in a glaucoma clinic
}

\author{
S. E. NORELL AND P.-A. GRANSTRÖM \\ From the Departments of Ophthalmology and Social Medicine, \\ Huddinge University Hospital, S-141 86 Huddinge, Sweden
}

SUMMARY A recording medication monitor and fluorescein technique were used to study selfmedication by 82 patients with primary open-angle glaucoma for whom pilocarpine eye drops 3 times a day had been prescribed. Of these patients $34(41 \%)$ missed 6 or more doses during a 20-day period, and for $35(43 \%)$ an 8-hour dose interval was exceeded at least $20 \%$ of the time. Consequences may include lack of effectiveness in preventing visual loss and unnecessary prescription of more potent, and more toxic, drugs.

About $50 \%$ of patients on long-term medication do not take their drugs as prescribed (Sackett and Haynes, 1976). This may be one of the major reasons for failure of treatment in such conditions as hypertension (Sackett et al., 1975), heart failure (Johnston et al., 1978), and tuberculosis (Dixon et al., 1957). We have reviewed the development of visual field defects over a 2-year period among 40 patients with primary open-angle glaucoma treated at the eye clinic of our hospital. Of these patients $18(45 \%)$ have shown marked progression of their visual field defects despite continuous prescription of drug treatment.

Primary open-angle glaucoma often causes only marginal complaints but requires long-term and frequently inconvenient treatment. Treatment is aimed at preventing long-range damage and provides no subjective improvement to prove its benefit to the patient. In fact, drug treatment may even produce temporary symptoms, such as smarting or blurred vision. Nevertheless patients are expected to take their medication regularly. Moreover, taking the drugs regularly may be of particular importance in glaucoma treatment since the drugs commonly used, such as pilocarpine, have a relatively short duration of action (Ellis, 1977). Selfmedication has been called 'the most overlooked aspect of glaucoma' (Riffenburgh, 1966).

Some interview data are available on the medication behaviour of glaucoma patients. Vincent (1972) interviewed 62 glaucoma patients and 36

Correspondence to Dr S. E. Norell, Department of Social Medicine, Huddinge University Hospital, S-141 86 Huddinge, Sweden.
$(58 \%)$ said they failed to take their eye drops more often than once a month. In a study of 40 patients with chronic simple glaucoma interviews indicated that $11(28 \%)$ missed properly taking their medication more often than once a week (Bloch et al., 1977). In another study 94 glaucoma patients were asked, 'Why don't you take the eye drops exactly as the doctor said?' and $31 \%$ 'admitted incorrect usage of medications' (Spaeth, 1970). The phrasing of the questions in these studies and some of the comments made by the authors point out the difficulties in obtaining accurate information on medication behaviour by interview. Indeed, striking discrepancies have been found between interviews and more objective data on drug taking by patients on different kinds of medication (Bergman and Werner, 1963; Gordis et al., 1969; Roth and Caron, 1978). In ophthalmology, however, there is little objective information on medication behaviour. Self-medication of 2 patients with glaucoma using a medication monitor technique has been described (Yee et al., 1974). The purpose of the present study was to describe medication behaviour with pilocarpine eye drops among patients with primary openangle glaucoma treated in an eye clinic.

\section{Material and methods}

STUDY SAMPLE

Included in this study were all patients treated at the eye clinic of Huddinge University Hospital by 1 March 1977 who fulfilled the following 6 criteria in their medical records: (1) diagnosed as primary open-angle glaucoma with (2) glaucomatous visual field defects, (3) glaucomatous cupping of the optic 
disc, and (4) intraocular pressure above or equal to $21 \mathrm{mmHg}$ recorded at least twice in the same eye; (5) prescription of $4 \%$ pilocarpine eye drops 3 times a day, and (6) visual acuity of at least $2 / 60$ in a pilocarpine treated eye.

Of the 92 patients fulfilling these criteria 10 were lost before they were studied. (Data were collected between March 1977 and November 1978.) One patient was lost because he moved abroad, 3 because they died, 3 because their therapy was changed, and 3 for psychological or psychiatric reasons. The latter 3 refused to visit the clinic and had been out of eye drops for long periods of time during the past years. Medication behaviour of the remaining 82 patients ( 45 men and 37 women) was studied. Their ages varied between 56 and 90 (median 73) years.

\section{MEASUREMENT METHODS}

Self-administration of pilocarpine eye drops was studied with a medication monitor and fluorescein technique. These methods have been described and discussed elsewhere (Norell et al., 1979).

The medication monitor consists of a small plastic box with a holder for a $25 \mathrm{ml}$ medication bottle. An elastic flap linked to a microswitch signals to the electronic part of the monitor whether the eyedropper cap is on or off. The information on whether or not the bottle has been opened during the last hour is transferred to a Random Access Memory with a capacity of 511 hours. By connecting a separate read-out device an electrocardiographic recorder can be used to display a record of the total register content together with a time signal.

The monitor recorded the date and hour each time the medication bottle was opened during a 3-week period between visits to the eye clinic. The days of clinic visits were excluded, leaving a monitor record of $\mathbf{2 0}$ days for each patient. For $\mathbf{8}$ patients a monitor record was obtained only after a second 20-day period owing to defective monitor batteries during the first test period. The patients were not told the purpose of the monitor until the collection of data was completed. Informed consent was obtained from the patient before the monitor record was printed out by computer and analysed, and the procedures were approved by the committee on ethics. To control other sources of the drug all prescriptions and bottles of pilocarpine were recalled from the patient when he received the monitor. No further intervention in the patient's routine was made until the monitor data were collected.

A fluorescein technique was used to study the ability of patients to administer the eye drops into the conjunctival sac. Each patient was asked to apply eye drops, containing $0.04 \%$ fluorescein, 5 different times in each eye, giving 10 tests of whether or not these drops actually fell into the conjunctival sac. Of the 82 patients studied, 15 patients, according to interview, always had their pilocarpine eye drops administered by somebody else ( 5 by a nurse and 10 by relatives). These patients were not examined with the fluorescein technique. Another 3 patients were lost to the study before they were tested with the fluorescein technique.

\section{MEASURES OF MEDICATION BEHAVIOUR}

The intervals between doses is a fundamental measure of medication behaviour, which in turn depends on the number of doses taken as well as the spacing between doses. The monitor records provide information on the length of the intervals between openings of the medication bottle. Such intervals will represent dose intervals under certain conditions (Norell et al., 1979). Monitor data, therefore, may be presented as a frequency distribution of dose intervals, as in Table 1 , where $\mathrm{x}$ is the length of dose intervals in hours. If we choose arbitrarily any specific interval length of $t$ hours, then the sum of $(x-t)$ for all intervals where $x>t$ will give the total time exceeding the $t$ hours dose interval during the observation period. Dividing this sum by the length of the observation period, gives the proportion of time exceeding the $t$ hours dose interval.

This may also be illustrated graphically in a cumulative frequency curve based on the same monitor data (Fig. 1). A vertical line representing a certain interval length $(t)$, such as the line $t=8$ in Fig. 1, will divide the area over the curve into 2 parts, $\mathrm{A}$ and $\mathrm{B}$. The proportion of time exceeding $t$ hours dose interval is equal to $\mathrm{A} / \mathrm{A}+\mathrm{B}$. This proportion may be determined for $t=$ the drug's duration of action, thus estimating the proportion of the time when there is no drug effect. It may also be determined for $t=24 / n$, where $n$ is the number of daily doses prescribed, thus estimating the proportion of time exceeding the duration of dose intervals expected if all prescribed doses had been taken at equal intervals. Since in this study all patients had a prescription of pilocarpine eye drops 3 times a day, the proportion of time exceeding 8-hour dose interval was estimated.

Monitor data were also analysed in relation to the number of daily doses prescribed. For each patient there was an interval during the night (between 0100 and 0400) when no doses were taken. For each day (between 2 such intervals) the number of doses taken (d) was compared to the prescribed 3 doses a day. If $d<3$, then $(3-d)$ is the number of missed doses that day. If $\mathrm{d}>3$, then 
(d-3) is the number of extra doses that day. The number of missed doses during the 20-day period was calculated for each patient and divided by the number of doses prescribed for that period $(20 \times 3=$ 60). This gives the proportion of missed doses for each patient. Similarly the number of extra doses was calculated.

\section{Results}

One patient had $8 / 10$ positive fluorescein tests. All other patients examined with the fluorescein technique had $10 / 10$ positive tests, indicating that they themselves were able to administer the eye drops into the conjunctival sac. This somewhat unexpected finding may reflect their experience in taking eye drops after 0.5 to 23 (median 4.4 ) years of glaucoma treatment.

Monitor data are given in Table 1 as the frequency distribution of the duration of intervals between doses in hours for the patient sample. Fig. 1 is a cumulative frequency curve from Table 1 . Intervals between doses varied from 1 to 164 hours with a median of 8 hours and a maximum frequency at 6 hours. Of the 4542 dose intervals $840(18 \%)$ had a duration of 12 hours or more and $509(11 \%)$ had a duration of 4 hours or less.

For the study sample the proportion of time exceeding the $t$ hours dose interval was $21 \%$ for $\mathrm{t}=8$ (that is, $21 \%$ of the observed time was more than 8 hours from a previous dose), $11 \%$ for $t=10$, $6 \%$ for $\mathrm{t}=12$, and $4 \%$ for $\mathrm{t}=14$. Fig. 2 shows the proportion of time exceeding 8 hours dose interval for each patient in the study sample. This proportion varied from $3 \%$ to $88 \%$ with a median

Table 1 Frequency distribution of dose intervals in hours for the study sample $(n=82)$ during 20 days' medication

\begin{tabular}{rrrrrr}
\hline$x$ & \multicolumn{1}{r}{$f$} & $x$ & $f$ & $x$ & $f$ \\
\hline 1 & 8 & 15 & 65 & 32 & 3 \\
2 & 73 & 16 & 61 & 33 & 1 \\
3 & 139 & 17 & 49 & 34 & 1 \\
4 & 289 & 18 & 28 & 35 & 1 \\
5 & 352 & 19 & 11 & 43 & 1 \\
6 & 663 & 20 & 11 & 47 & 1 \\
7 & 555 & 21 & 6 & 48 & 4 \\
8 & 591 & 22 & 6 & 60 & 1 \\
9 & 403 & 23 & 6 & 88 & 1 \\
10 & 327 & 24 & 14 & 107 & 1 \\
11 & 302 & 25 & 4 & 121 & 1 \\
12 & 289 & 26 & 7 & 123 & 1 \\
13 & 168 & 27 & 1 & 152 & 1 \\
14 & 93 & 28 & 2 & 164 & 1 \\
\hline
\end{tabular}

$x=$ Length of intervals between doses (in hours). $f=$ Number of dose intervals recorded by the monitor. of $17 \cdot 2$. For $35(43 \%)$ of the 82 patients at least $20 \%$ of time was more than 8 hours distant from a previous dose.

In Fig. 3 the proportion of missed doses is given for each patient, which varied from 0 to $83 \%$ with

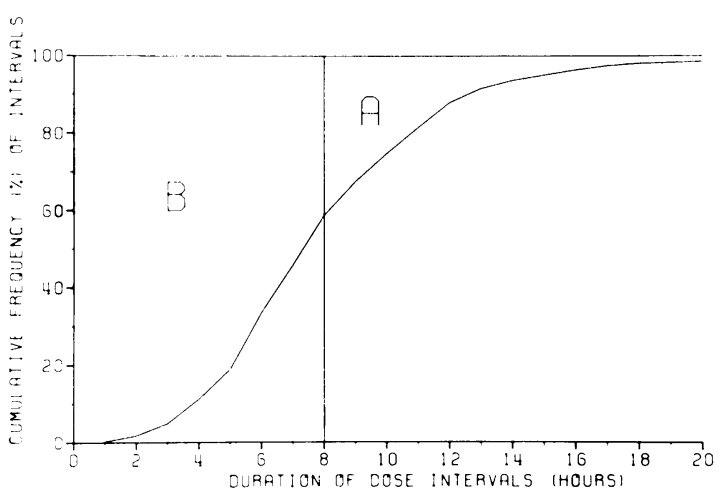

Fig. 1 Cumulative frequency distribution of dose intervals; from Table 1 (see text)

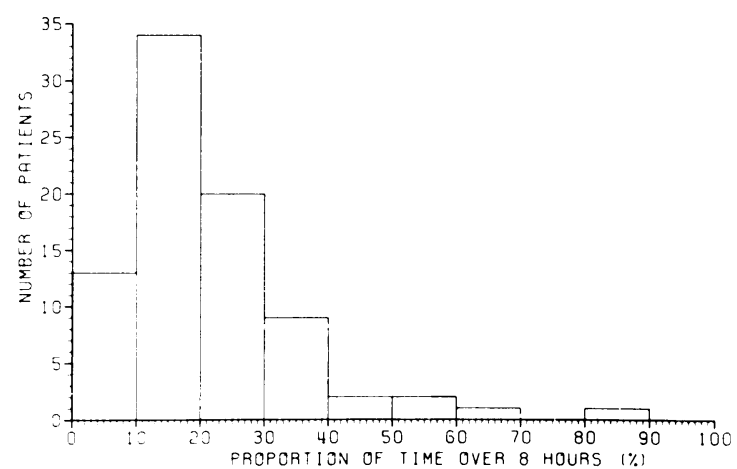

Fig. 2 Proportion of time exceeding 8-hour dose interval for each patient in the study sample $(n=82)$

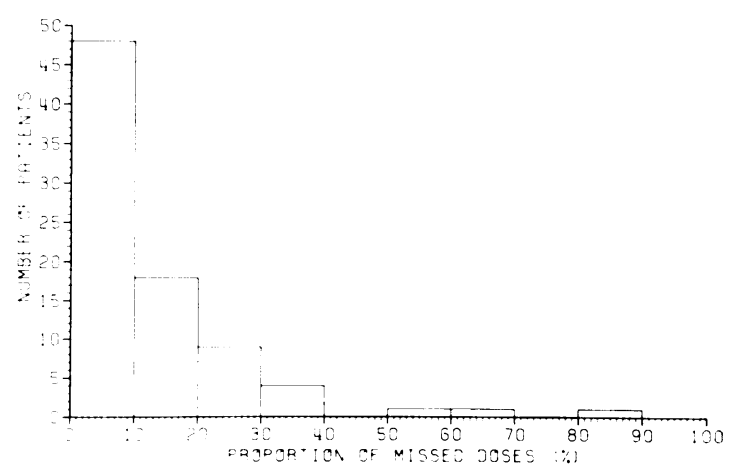

Fig. 3 Proportion of missed doses for each patient in the study sample $(n=82)$. Of the 48 patients who missed less than $10 \%$ of the doses prescribed 21 missed no doses and 43 missed less than $5 \%$ of doses 
a median of $3.3 \%$. Of the 82 patients studied 34 $(41 \%)$ omitted at least $10 \%$ and $16(20 \%)$ omitted at least $20 \%$ of the prescribed doses. The total number of missed doses in the study sample was 507 , or $10 \%$ of the doses prescribed. Similarly, the total number of extra doses was 50 , or $1 \%$ of the doses prescribed, varying between 0 and $12 \%$ for individual patients.

The proportion of time exceeding the 8-hour dose interval is determined not only by the number of doses taken but also by the spacing between doses. This is evident in Table 2, where the proportion of missed doses is related to the proportion of time exceeding the 8-hour dose interval. For example, among the 21 patients taking all the prescribed doses, 14 exceeded the 8-hour dose interval at least $10 \%$ of the time owing to irregular spacing between doses.

\section{Discussion}

Some problems in the measurement of medication behaviour were discussed in a previous paper (Norell et al., 1979). It was concluded that the medication monitor and fluorescein technique offer more accurate and detailed information on medication behaviour than, for example, similar data obtained by interview.

The effect of drug treatment is related not only to the number of doses taken but also to the spacing between doses. Finding a measure which takes both these factors into account is a major problem in describing medication behaviour. The proportion of time exceeding a $t$ hours dose interval offers a solution to this problem. It has been suggested that a single dose of pilocarpine is effective in lowering intraocular pressure for about 8 hours or less (Ellis, 1977; Heilmann and Richardson, 1978), while some data indicate a longer duration of action (Drance et al., 1974; Quigley and Pollack, 1977). On the assumption that the effective duration is about 8 hours, it would seem reasonable to consider the proportion of time more than 8 hours from a

Table 2 Missed doses in relation to time exceeding 8 -hour dose interval for each patient in the study sample

\begin{tabular}{lrrrrl}
\hline \multirow{7}{*}{$\begin{array}{l}\text { Time over } \\
8 \text { hours }(\%)\end{array}$} & \multicolumn{1}{l}{ Missed doses $(\%)$} & & \\
\cline { 2 - 6 } & 0 & $1-$ & $10-$ & $20-$ & Total \\
\hline $0-$ & 7 & 6 & 0 & 0 & 13 \\
$10-$ & 12 & 17 & 5 & 0 & 34 \\
$20-$ & 2 & 2 & 10 & 6 & 20 \\
$30-$ & 0 & 2 & 3 & 10 & 15 \\
Total & 21 & 27 & 18 & 16 & 82 \\
\hline
\end{tabular}

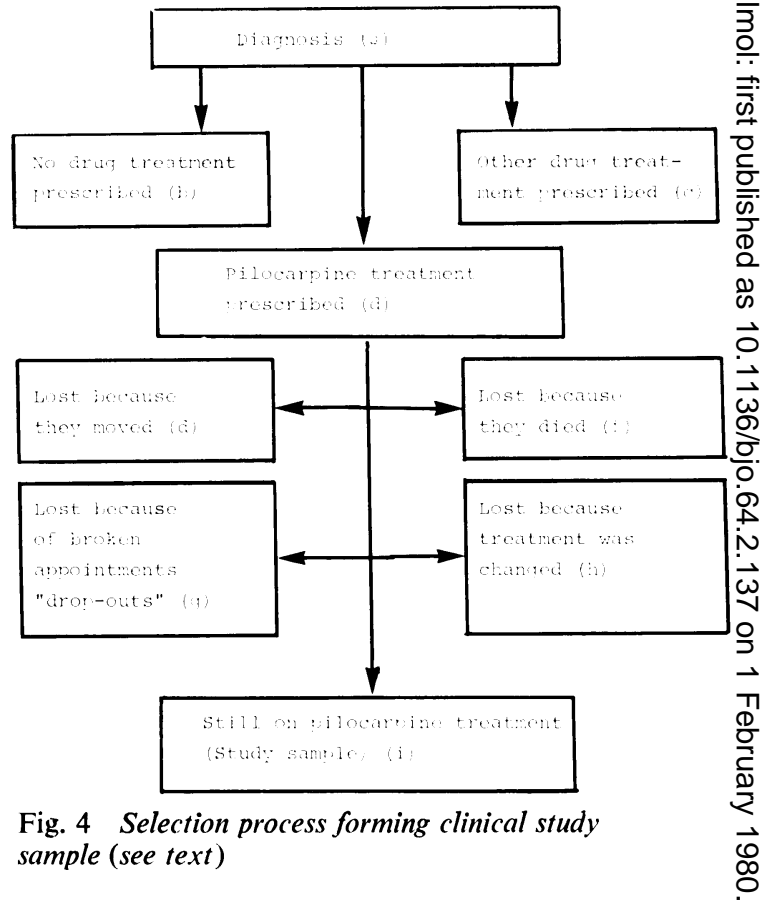

previous dose as a rough estimate of the proportion of time when there is no drug effect. However, a more accurate description of the time without drug effect would have to take several factors into account. $\frac{0}{8}$ First, drug effect is not an all-or-nothing pheno- $\stackrel{\varrho}{\square}$ menon, and the effect of a single dose will decrease $\overrightarrow{\overrightarrow{0}}$ gradually over time. Secondly, if several doses are $\exists$ taken with shorter intervals, say 1 or 2 hours, the concentration of the drug in the anterior chamber of the eye may rise above the concentration followed $\frac{0}{0}$. by a single dose, and this may give a longer 'duration' after the last dose. Thirdly, if the level of the intra- 3 . ocular pressure (IOP) is important as a cause of $\delta$ visual loss in glaucoma, and if, for example, IOP is higher during the morning hours than later 0 during the day, then it may be more important to 'cover' these hours. A further refinement of the measure used here will have to take these and possibly other factors into consideration.

From the patients diagnosed and for whom pilo- $N$ carpine was initially prescribed the study sample was selected by the process summarised in Fig. 4. After diagnosis (a) pilocarpine treatment is pre- 0 scribed for some patients (d) but not for others (b, $\underset{\Phi}{\overparen{D}}$ c). Of the patients for whom pilocarpine treatment $\stackrel{\oplus}{?}$ was prescribed some will have their therapy changed 0 to other drugs, for example, phospholine iodide, or operation (h). In this study 3 patients were lost $\stackrel{\mathbb{Q}}{\mathbb{Q}}$ because their treatment was changed between $\stackrel{\mathbb{Q}}{\propto}$ 1 March 1977 and their entry into the study (median 
time about 10 months). Over many years a considerable proportion of patients initially on pilocarpine have their treatment changed. Since the reason for this may be that the pilocarpine treatment did not work, an overrepresentation of patients not adherent to the prescribed regimen should be expected in this group. Other patients will be lost because they have not maintained follow-up appointments $(\mathrm{g})$. In this study 3 patients were lost during the time before their entry into the study. For long periods of time they did not visit the clinic, had no supply of pilocarpine, and were obviously not taking the drug. Other studies have shown that $37 \%$ of patients with glaucoma or ocular hypertension did not return for follow-up appointments during 12 to 20 months after initial diagnosis (Bigger, 1976) and that among patients referred for further testing from a glaucoma screening programme $20 \%$ did not keep their appointment (Rosenthal, 1967).

The selection process forming a group for clinical study is of course more complicated than it would appear from the diagram. In any case a considerable proportion of the patients for whom pilocarpine was initially prescribed were lost in the process. Furthermore, an overrepresentation of patients not adherent to the prescribed regimen would be expected among those lost from the sample selected for study. A follow-up study of an inception cohort, that is, patients for whom pilocarpine was initially prescribed from a well defined population, might clarify this situation.

Our findings suggest that missed doses are more frequent than has been found in other studies of medication behaviour in glaucoma (Spaeth, 1970; Vincent, 1972; Bloch et al., 1977). This is not surprising, since these studies have been based on interview data, with underreporting of missed doses as a major problem (Bergman and Werner, 1963; Gordis et al., 1969; Roth and Caron, 1978). In addition, our data show that dose intervals exceeding the expected interval length will often be due to irregular spacing between doses rather than omitted doses. The proportion of time exceeding an 8-hour dose interval varied widely between patients, from $3 \%$ to $88 \%$, with a median of $17 \cdot 2 \%$. Furthermore, the study sample could be expected to be more adherent to the prescribed regimen than the patients for whom pilocarpine was initially prescribed.

Visual loss from glaucoma during drug treatment is often dealt with by prescribing more potent, and more toxic, drugs. This may not be appropriate, since lack of effect in preventing visual loss may be due to irregular drug taking rather than that the drug is not effective. Similarly, the outcome of clinical drug trials may be influenced by the patients' medication behaviour. This calls for simple methods to check that patients take their drugs regularly, and useful strategies to improve medication behaviour. These problems are under investigation in our group.

Supported in part by grants from the National Corporation of Swedish Pharmacies, ANIS (Council for Information and Drug Statistics).

\section{References}

Bergman, A. B., and Werner, R. J. (1963). Failure of children to receive penicillin by mouth. New England Journal of Medicine, 268, 1334-1338.

Bigger, J. F. (1976). A comparison of patient compliance in treated vs untreated ocular hypertension. Transactions of the American Academy of Ophthalmology and Otolaryngology, 81, 277-285.

Bloch, S., Rosenthal, A. R., Friedman, L., and Caldarolla, P. (1977). Patient compliance in glaucoma. British Journal of Ophthalmology, 61, 531-534.

Dixon, W. M., Stradling, P., and Wootton, I. D. P. (1957). Outpatient P.A.S. therapy. Lancet, 2, 871-872.

Drance, S. M., Bensted, M., and Schulzer, M. (1974). Pilocarpine and intraocular pressure: duration of effectiveness of $4 \%$ and $8 \%$ pilocarpine instillation. Archives of Ophthalmology, 91, 104-106.

Ellis, P. (1977). Ocular Therapeutics and Pharmacology, 5th edition, pp. 128-129. C. V. Mosby: St Louis.

Gordis, L., Markowitz, M., and Lilienfeld, A. M. (1969). The inaccuracy in using interviews to estimate patient reliability in taking medications at home. Medical Care, 7, 49-54.

Heilmann, K., and Richardson, K., eds. (1978). Glaucoma, Conceptions of a Disease, p. 272. Georg Thieme: Stuttgart.

Johnston, G. D., Kelly, J. G., and McDevitt, D. G. (1978). Do patients take digoxin? British Heart Journal, 40, 1-7.

Norell, S., Granström, P.-A., and Wassén, R. (1979). A medication monitor and fluorescein technique designed to study medication behaviour. Acta Ophthalmologica, in press.

Quigley, H. A., and Pollack, I. P. (1977). Intraocular pressure control with twice-daily pilocarpine in two vehicle solutions. Annals of Ophthalmology, 9, 427-430.

Riffenburgh, R. (1966). Doctor-patient relationship in glaucoma therapy. Archives of Ophthalmology, 75, 204-206.

Rosenthal, J. (1967). Evaluation of recall system for glaucoma suspects and patients. Sight Saving Review, 37, 78-82.

Roth, H. P., and Caron, H. S. (1978). Accuracy of doctors' estimates and patients' statements on adherence to a drug regimen. Clinical Pharmacology and Therapeutics, 23, 361-370.

Sackett, D. L., Haynes, R. B., Gibson, E. S., Hackett, B. C., Taylor, D. W., Roberts, R. S., and Johnson, A. L. (1975). Randomised clinical trial of strategies for improving medication compliance in primary hypertension. Lancet 1, 1205-1207.

Sackett, D. L., and Haynes, R. B., eds. (1976). Compliance with Therapeutic Regimens. Johns Hopkins University Press: Baltimore.

Spaeth, G. L. (1970). Visual loss in a glaucoma clinic. I. Sociological considerations. Investigative Ophthalmology, 9, 73-82.

Vincent, P. (1972). Patients' viewpoint of glaucoma therapy. Sight Saving Review, 42, 213-221.

Yee, R. D., Hahn, P. M., and Christensen, R. E. (1974). Medication monitor for ophthalmology. American Journal of Ophthalmology, 78, 774-778. 